\title{
The Occurrence of Acalculous Cholecystitis in a Drug Hypersensitivity Reaction: A Case Report
}

\author{
Noviana Joenputri ${ }^{1}$, Ketut Suryana ${ }^{2}$ \\ ${ }^{1}$ General Practitioner, Department of Internal Medicine, Wangaya General Hospital, Denpasar, Bali, Indonesia \\ ${ }^{2}$ Immunologist, Department of Internal Medicine, Wangaya General Hospital, Denpasar, Bali, Indonesia
}

\begin{abstract}
Acalculous cholecystitis (AC) is defined as necro-inflammatory disease in gallbladder in the absence of gallstone which is caused by multifactorial. This case report aimed to show a severe systemic drug allergic reaction which attributed to AC. We reported a 23-year-old male who experienced acute systemic hypersensitivity reaction after 12 hours taking paracetamol. The patient showing generalized urticaria, epigastric and right upper quadrant of abdominal pain. Laboratory findings showed leukocytosis. Abdominal ultrasound showed the thickening of gallbladder wall, without the presence of gallstone and widening of bile duct. The patient was suspected of having vasculitis which complicated to gallbladder ischemia as underlying cause of AC.
\end{abstract}

Keywords: acalculous cholecystitis, drug allergy, hypersensitivity reaction

\section{Introduction}

Acalculous cholecystitis (AC) is a rare and life-threatening disease. [1, 2] $\mathrm{AC}$ is defined as a necro-inflammatory disease in gallbladder in the absence of gallstones. The pathogenesis of $\mathrm{AC}$ is multifactorial. [3, 4] One of the underlying pathogens of $\mathrm{AC}$ is ischemia which can be caused by vasculitis in hypersensitivity reaction. [3-6] Vasculitis causes damage to blood vessels both venules and arterioles. It disturbs the perfusion of the affected organ. We reported a rare case of $\mathrm{AC}$ in patient who experienced hypersensitivity reaction to paracetamol.

\section{Case Illustration}

A 23-year-old man came to the Emergency Department with complaints of generalized body rash and itchy twelve hours after consuming paracetamol. The patient also complained of epigastric and right upper quadrant abdominal pain that appeared together with the rash. There were no factors that aggravate or alleviate the abdominal pain. The patient also complained of nausea and vomiting. Abdominal pain felt with VAS 4/10. Complaints of fever, shortness of breath, diarrheas were denied. The patient had taken paracetamol several times but never experienced abnormal reaction. The patient had experienced allergic reaction two times, but the causes were not identified. Histories of asthma, food allergies or drug allergies were denied. His mother and sister had history of asthma.

Vital signs were in normal limits. Epigastric and right upper quadrant of abdominal pain was found in abdominal palpation. There were generalized papular and annular urticarial plaques (Figure 1). Laboratory findings found leukocytosis $(16,570 / \mathrm{uL})$, neutrophilia $(83.8 \%)$, and normal eosinophil level $(0.2 \%)$.

The patient was diagnosed with anaphylaxis reaction due to paracetamol allergic. Paracetamol treatment was immediately stopped. The patient was given intravenous fluid, intramuscular epinephrine injection, intravenous esomeprazole $1 \times 40 \mathrm{mg}$, and oral loratadine $2 \times 10 \mathrm{mg}$. The patient was observed for the vital signs and symptoms. Abdominal pain and urticaria in this patient persist after 24 hours of observation.
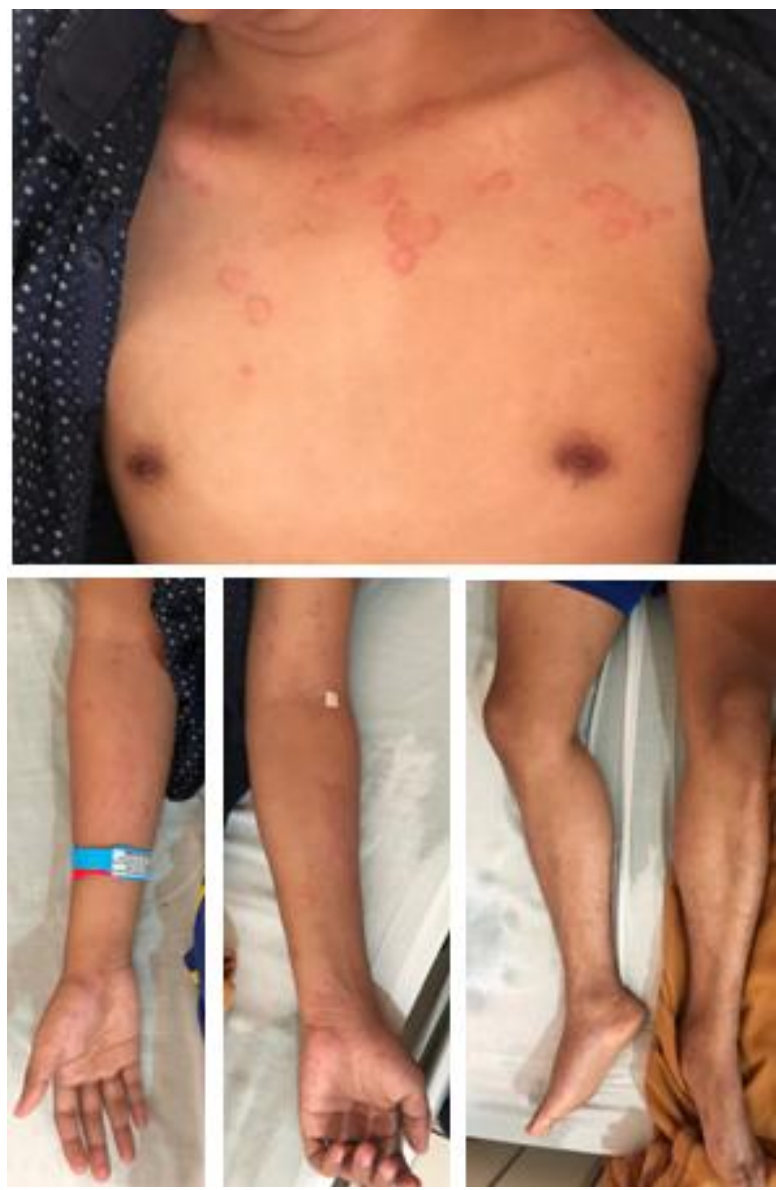

Figure 1: The presentation of cutaneous manifestation

The patient suspected of having vasculitis. We underwent abdominal ultrasound examination to evaluate of abdominal pain. Abdominal ultrasound revealed the thickening of gallbladder wall, no gallstone, and no widening of bile duct. 


\section{International Journal of Science and Research (IJSR) \\ ISSN: 2319-7064}

ResearchGate Impact Factor (2018): 0.28 | SJIF (2019): 7.583

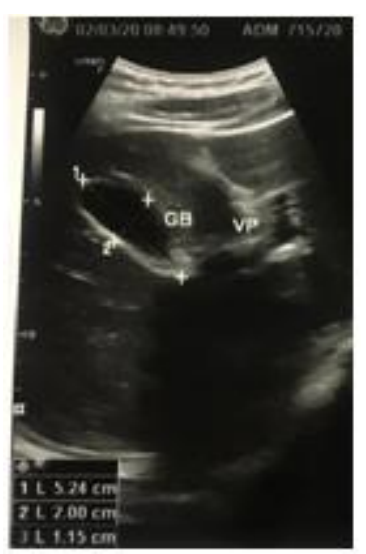

Figure 2: Abdominal ultrasound showed the thickening of gallbladder wall, no gallstone, and no widening of bile duct.

The patient was diagnosed with acute $\mathrm{AC}$ and vasculitis. Patients were given additional therapy such as intravenous methylprednisolone $2 \times 62.5 \mathrm{mg}$ and intravenous antibiotic to treat acute AC. The patient was treated for eight days, the he was discharged after symptoms improved.

\section{Discussions}

The patient in this case, a 23-year-old male, was initially diagnosed with anaphylaxis reaction due to paracetamol allergy. This was based on the history of taking paracetamol 12 hours before the onset of cutaneous and gastrointestinal symptoms. A few numbers of anaphylaxis reactions due to paracetamol allergy have been reported by Khan et al, only $0.8 \%$. [7] Most patients with anaphylaxis showing cutaneous (76.7\%), cardiac (68.9\%), and gastrointestinal (64.3\%) manifestations. [7] The most gastrointestinal manifestation occurred were vomiting (13.2\%) and abdominal pain (8.5\%). [7] The patient was treated according to the guideline for management of anaphylaxis reaction. [8, 9] The main therapy for anaphylaxis is administration of intramuscular epinephrine.[8, 9] Second generation $\mathrm{H} 1$-antihistamine and proton-pump inhibitor were given only for symptomatic management.

The vital signs were stable during 24 hours of observation. However, the symptoms of urticaria and abdominal pain still persist. Urticaria in allergic reaction generally disappeared within 24 hours. If the urticaria lesion persists for more than 24 hours, this condition must be considered as vasculitis as a pathogenesis of the urticarial reaction. [10, 11] Urticarial vasculitis showed the episode of recurrent urticarial lesion and it usuallyaccompanied by other systematic disorders. $[12,13]$

Vasculitis shows an inflammation of the blood vessel walls caused by a various of non-specific reaction, one of which is hypersensitivity reaction. [10] Some evidence showed the participation of small blood vessel in the allergic reaction, as well as the understanding that some clinical symptoms that cannot be explained, may be due to allergic. [10] The presence of sensitizing antigens causes arteriolar constriction and stoppage of circulation. Leukocytes attached to the endothelium of blood vessels and migrated through capillary walls and venules in large quantities. In addition, some leukocytes are agglutinated causing emboli that block some capillaries and venules. Drugs, invective agents, antibiotics, and malignant tumors act as antigens. Vascular damage is probably the result of antigens and antibodies reaction in the vessel wall. [10] To confirm the type of vasculitis, a skin biopsy is needed, but it was not done in this case due to limited facilities.

Abdominal ultrasound results obtained thickening and edema in the gallbladder wall without stones and bile duct dilatation. In this patient, there was AC. Acute AC is defined as an acute necro-inflammatory disease of the gallbladder in the absence of gallstones and has a multifactorial pathogenesis. [1, 3, 4] Gallstones were the main cause of acute cholecystitis, non-gallstones or AC occurs for only approximately $10 \%$ of acute cholecystitis, hence, it is often overlooked and leading to a delayed diagnosis that increase the morbidity and mortality than calculous cholecystitis. [6, $14,15]$ Ischemia plays an important role in the pathogenesis of AC. [5]

Gallbladder has a terminal artery. If visceral hypoperfusion occurs due to various things, there is a decrease in gallbladder perfusion and results in gallbladdermucosal ischemia. [6] Halaka et al reported differences in capillary flow in calculous cholecystitis and AC. In calculous cholecystitis, capillary flow appears regular, but in contrast capillary flow is less and irregular in AC. [16] Therefore, AC is also referred as 'acute ischemic cholecystitis'. Ischemia that occurs in the gallbladder mucosa can cause hypomotility resulting in gallbladder stasis that causes intraluminal exposure of the gallbladder wall, ultimately can cause ischemia, inflammation, and potentially necrosis. In addition, if ischemia, inflammation, or infection occurs continuously, it can cause perforation. [6, 17] AC is more dangerous because of the high potential for necrosis and perforation, especially in late diagnosis.

Actually, there are no specific criteria for diagnosing AC. $[15,17]$ However, a study stated that the diagnosis of AC was usually based on radiological results. Ultrasonography has been used as first choice to evaluate suspected acute AC because of its advantages. [1]In this case, diagnosis of AC was established based on the presence of 3 major criteria such as thickening of gallbladder wall $>3 \mathrm{~mm}$, edema of the gallbladder wall, sonography murphy sign; and one minorcriteriasuch as gall bladder distension $5.24 \mathrm{~cm}(>5 \mathrm{~cm}$ in transverse diameter) on ultrasound examination. [18] The presence of leukocytosis $>10,000 / \mathrm{uL}$ also supported the diagnosis of AC. [17, 19]

It can be concluded that hypersensitivity to the drug can be an unknown factor in some patients with $\mathrm{AC}$. The patient in this case most likely suffered from AC since he was first admitted to the hospital because of a hypersensitivity reaction caused by paracetamol. Some of the supporting reasons were: (1) the patient had just taken paracetamol 12 hours before the initial episodes of rash, epigastric and right upper quadrant abdominal pain appeared, (2) manifestation of rash and abdominal pain persisted more than 24 hours after initial treatment, (3) abdominal ultrasound and laboratory findings supported AC. 


\section{International Journal of Science and Research (IJSR) \\ ISSN: 2319-7064}

ResearchGate Impact Factor (2018): 0.28 | SJIF (2019): 7.583

The presence of persistent urticaria, the recent use of paracetamol, and the development of AC allowed us to consider that $\mathrm{AC}$ in this patient was related to vasculitis due to paracetamol-induced hypersensitivity reactions. Vasculitis causes gallbladder blood flow disruption leading to gallbladder ischemia which plays an important role in pathogenesis of AC.

In this case, thereof most likely causative factor of $\mathrm{AC}$ is drug hypersensitivity. Therefore, the suspected drug must be stopped and clinical improvement should be observed. The main treatment is to eliminate the causative factors, so the patient does not need surgery. [10, 20, 21] The treatment of vasculitis is corticosteroid because it has a specific effect on vascular lesion and helps healing and sclerosis. [10]

Second-generationH1-antihistamines are the first-line treatment for acute urticaria with a good side effect profile. [22] The administration of intravenous antibiotics plays a major role in the treatment of AC in hospital setting. If the infection is mild-moderate, antibiotics such as cefazolin, cefuroxime, and ceftriaxone are preferred. [14]Therefore, in this case, we provide therapy for vasculitis in the form of intravenous methylprednisolone and second-generation of H1-antihistamine, loratadine. Antibiotic was given to treat AC.

\section{Conclusion}

The present report underlines the importance of assessing patients who have systemic hypersensitivity reaction comprehensively. Vasculitis due to drug-induced hypersensitivity reactions can cause organ ischemia that leads to acute $\mathrm{AC}$ which is dangerous because of its high potential for necrosis and perforation. The clinicians should be aware for the occurrence of AC in patients with systemic hypersensitivity reaction. Early diagnosis and prompt treatment can decrease the morbidity and mortality in $\mathrm{AC}$ patients.

\section{References}

[1] Barie PS, Eachempati SR. Acute acalculous cholecystitis. Current gastroenterology reports. 2003 Aug 1;5(4):302-9.

[2] Owen CC, Jain R. Acute acalculous cholecystitis. Current treatment options in gastroenterology. 2005 Apr 1;8(2):99.

[3] Glenn FR, Becker CG. Acute acalculous cholecystitis. An increasing entity. Annals of surgery. 1982 Feb;195(2):131.

[4] Johanning JM, Gruenberg JC, Roty Jr AR, Hill MN, Diebel LN. The changing face of cholecystectomy/Discussion. The American surgeon. 1998 Jul 1;64(7):643.

[5] Warren BL. Small vessel occlusion in acute acalculous cholecystitis. Surgery. 1992 Feb;111(2):163-8.

[6] Barie PS, Fischer E. Acute acalculous cholecystitis. Journal of the American College of Surgeons. 1995;180(2):232-44.

[7] Khan NU, Shakeel N, Makda A, Mallick AS, Ali Memon M, Hashmi SH, Khan UR, Razzak JA.
Anaphylaxis: incidence, presentation, causes and outcome in patients in a tertiary-care hospital in Karachi, Pakistan. QJM: An International Journal of Medicine. 2013 Dec 1;106(12):1095-101.

[8] Campbell RL, Li JT, Nicklas RA, Sadosty AT. Emergency department diagnosis and treatment of anaphylaxis: a practice parameter. Annals of Allergy, Asthma \& Immunology. 2014 Dec 1;113(6):599-608.

[9] Ring J, Beyer K, Biedermann T, Bircher A, Duda D, Fischer J, Friedrichs F, Fuchs T, Gieler U, Jakob T, Klimek L. Guideline for acute therapy and management of anaphylaxis. Allergo journal international. 2014 May 1;23(3):96-112.

[10] McCombs RP, Patterson JF, MacMahon HE. Syndromes associated with allergic vasculitis. New England Journal of Medicine. 1956 Aug 9;255(6):25161.

[11] Black AK. Unusual urticarias. The Journal of dermatology. 2001 Nov;28(11):632-4.

[12] Kambe N, Kitao A, Nishigori C, Miyachi Y. Latephase urticaria update. Current allergy and asthma reports. 2002 Jul 1;2(4):288-91.

[13] Tsunemi Y, Saeki H, Ihn H, Tamaki K. Recurrent cutaneous eosinophilic vasculitis presenting as annular urticarial plaques. ACTA DERMATOVENEREOLOGICA-STOCKHOLM-. 2005 Jan 1;85(4):380.

[14] Kalliafas S, Ziegler DW, Flancbaum L, Choban PS. Acute acalculous cholecystitis: incidence, risk factors, diagnosis, and outcome. The American Surgeon. 1998 May 1;64(5):471.

[15] Huffman JL, Schenker S. Acute acalculous cholecystitis: a review. Clinical Gastroenterology and Hepatology. 2010 Jan 1;8(1):15-22.

[16] Hakala T, Nuutinen PJ, Ruokonen ET, Alhava E. Microangiopathy in acute acalculous cholecystitis. Br J Surg 1997;84: 1249-52.

[17] Balmadrid B. Recent advances in management of acalculous cholecystitis. F1000Research. 2018;7.

[18] BariePS, Eachempati SR. Acute acalculous cholecystitis. Gastroenterology Clinics. 2010 Jun 1;39(2):343-57.

[19] Savoca PE, Longo WE, Zucker KA, McMillen MM, Modlin IM. The increasing prevalence of acalculous cholecystitis in outpatients. Results of a 7-year study. Annals of surgery. 1990 Apr;211(4):433.

[20] Michielsen PP, Fierens H, Van Maercke YM. Druginduced gallbladder disease. Drug safety. 1992 Jan $1 ; 7(1): 32-45$.

[21] Bryan B. Recent advances in management of acalculous cholecystitis. F1000Research. 2018;7.

[22] Schaefer P. Acute and chronic urticaria: evaluation and treatment. American family physician. 2017 Jun 1;95(11):717-24. 